\title{
Ações de enfermagem para a prevenção e controle do delirium em pacientes pós-operatório de cirurgia cardíaca: uma revisão integrativa
}

RESUMO | Objetivo: Analisar as ações de enfermagem no controle e prevenção do delirium em pacientes pós-operatório de cirurgia cardíaca apresentados na literatura. Método: Estudo de revisão integrativa da literatura. A busca e seleção dos artigos foi realizada através das bases de dados LILACS, MEDLINE, BDENF, IBECS, MEDCARIB, CUMED, Secretaria Estadual de Saúde de São Paulo e coleciona SUS. A amostra foi composta por nove estudos. Resultados: Identificamos entre as ações para o controle e prevenção do delirium a visita familiar estruturada, a comunicação, importância de realizar a orientação verbal sobre o tempo e espaço, reconhecer o delirium precocemente e utilizar escalas para o diagnóstico, sendo a CAM-ICU a mais recomendada e de melhor aplicação. Conclusão: A enfermagem é essencial para realizar ações preventivas e reconhecer o delirium, muitas ações de prevenção são realizadas e, a comunicação e a presença da família é fundamental para se prevenir essa patologia.

Palavras-chaves: Delirium; Cuidados de enfermagem; Procedimentos Cirúrgicos Cardíacos.

ABSTRACT | Objective: To analyze nursing actions in the control and prevention of delirium in patients in the postoperative period of cardiac surgery presented in the literature. Method: Study of integrative literature review. The search and selection of articles was carried out through the LILACS, MEDLINE, BDENF, IBECS, MEDCARIB, CUMED, São Paulo State Health Secretariat and SUS collections. The sample consisted of nine studies. Results: We identified among the actions for the control and prevention of delirium the structured family visit, communication, the importance of carrying out verbal orientations in time and space, recognizing the delirium early and using diagnostic scales, CAM-ICU being the most recommended and best application. Conclusion: Nursing is essential to carry out preventive actions and to recognize delirium, many preventive actions are carried out and communication and the presence of the family are essential to prevent this pathology.

Keywords: Delirium; Nursing Care; Cardiac Surgical Procedures.

RESUMEN | Objetivo: Analizar las acciones de enfermería en el control y prevención del delirio en pacientes en el postoperatorio de cirugía cardíaca presentadas en la literatura. Método: estudio de revisión integradora de la literatura. La búsqueda de los artículos se realizó a través de las bases de datos LILACS, MEDLINE, BDENF, IBECS, MEDCARIB, CUMED, Secretaría de Salud del Estado de São Paulo y recolecta SUS, la muestra estuvo compuesta por nueve estudios. Resultados: Entre las acciones para el control y prevención del delirio, identificamos la visita familiar estructurada, la comunicación, la importancia de realizar una guía verbal en tiempo y espacio, el reconocimiento temprano del delirio y el uso de escalas diagnósticas, siendo Se recomienda CAM-UCl y se aplica mejor. Conclusión: Enfermería es fundamental para realizar acciones preventivas y para reconocer el delirio, se realizan muchas acciones preventivas y la comunicación y la presencia de la familia son fundamentales para prevenir esta patología.

Palabras claves: Delirio; Atención de Enfermería; Procedimientos Quirúrgicos Cardíacos.

\section{Jéssica Thais Nunes de Oliveira}

Enfermeira Residente em Saúde cardiovascular do Hospital das Clínicas da Universidade Federal de Minas Gerais (HC-UFMG). Graduada em enfermagem pelo Centro Universidade de Belo Horizonte (UNIBH). ORCID: 0000-0001-8606-9954

\section{Karla Cordeiro Gonçalves}

Enfermeira do HC-UFMG. Mestre em Ciências Aplicadas à Saúde do Adulto pela UFMG. Especialista em cardiologia pelo Hospital das Clínicas da Faculdade de Medicina da Universidade de São Paulo (HC-FMUSP).

ORCID: 0000-0002-5123-9308

Recebido em: 30/12/2020

Aprovado em: 26/01/2021

\section{Salete Maria de Fatima Silqueira}

Enfermeira. Dra em Saúde Pública pela USP-Ribeirão Preto. Tutora da residência multiprofissional em Saúde Cardiovascular do HC-UFMG.

ORCID: 0000-0002-4248-7107

INTRODUÇÃO

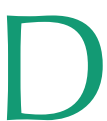
elirium é uma síndrome mental transitória de início súbito, desenvolvida em um curto período de tempo. Pode apresentar-se em três formas: hipoativa que se caracteriza por rebaixamento do nível de consciência, prostração e redução da comunicação. Hiperativa onde predomina a agitação, agressividade e até mesmo risco de auto e heteroagressão. $E$, a mista que apresenta os sintomas de ambas os polos ${ }^{(1,2)}$.

As causas neurológicas que levam ao aparecimento do delirium são pouco conhecidas, porém alguns fatores contribuem para o desenvolvimento desse quadro, sendo divididos em fatores predisponentes, entre eles estão a idade avançada, déficit cognitivo de base, uso de álcool e drogas, tabagismo, diabetes, insuficiência cardíaca e disfunção renal. Fatores precipitantes como o uso de medicamentos, tais como o opioides e benzodiazepínicos, manejo inadequado da dor, tempo prolongado de ventilação mecânica, distúrbios metabólicos e hidroeletrolíticos, infecção pós-operatória, sepse, contenção física, isolamento e ci- 
rurgias de grande porte como cardíaca, abdominal e de fêmur ${ }^{(3)}$.

A incidência do delirium em paciente pós-operatórios varia entre 10 a 60\%, tendo um aumento quando eles estão inseridos em uma unidade de terapia intensiva, principalmente se em uso de ventilação mecânica, aumentando cerca de $80 \%$ dos casos. Além disso, o tipo de procedimento cirúrgico também pode aumentar essa incidência, como no caso de cirurgias cardíacas onde a incidência dessa síndrome foi em mais de 51\%, devido ao maior número de comorbidades, função cognitiva mais baixa no pré-operatório ou problemas relacionados à própria cirurgia ${ }^{(3)}$.

A cirurgia cardíaca no Brasil é o segundo procedimento mais realizado, com estimativa de 102 mil cirurgias/ano ficando atrás somente dos Estados unidos, onde são realizadas cerca de 300 mil cirurgias/ ano. Esse aumento é consequente dos números de doenças cardiovasculares, que atualmente é a segunda patologia que mais causam óbitos no mundo ${ }^{(4)}$.

O delirium em pós-operatório de cirurgia cardíaca é uma complicação que aparece cada vez mais, a incidência de complicações neurológicas nesse tipo de procedimento pode variar 0,4 a $80 \%$, entre os tipos de cirurgias, a revascularização do miocárdio e as trocas valvares são as que mais apresentam alterações neurológicas. Atualmente a etiologia do delirium em cirurgias cardíacas não são bem claras, porém alguns estudos relacionam o aparecimento dessa síndrome pelo tempo de circulação extracorpórea (CEC), devido as alterações que podem ser geradas nos neurotransmissores ${ }^{(5,6)}$.

O delirium no pós-operatório pode causar complicações diversas no paciente, entre elas o aumento da mortalidade e tempo de ventilação mecânica, distúrbios cognitivos de longo prazo e mais tempo de internação em terapia intensiva. Muitas vezes o reconhecimento e o diagnóstico de delirium não são realizados devido ao paciente estar intubado, além do que, em algumas instituições, não têm instrumentos para que seja realizado o reconhecimento precoce pela equipe de enfermagem e multiprofissional ${ }^{(2)}$.

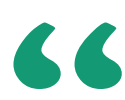

\section{A cirurgia cardíaca no Brasil é o segundo procedimento mais realizado, com estimativa de 102 mil cirurgias/ ano ficando atrás somente dos Estados unidos, onde são realizadas cerca de 300 mil cirurgias/} ano.

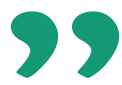

A equipe de enfermagem tem o papel fundamental no controle e reconhecimento do delirium no pós operatório de cirurgia cardíaca, é uma das primeiras a poder reconhecer a presença do delirium, pois as alterações que o paciente pode apresentar é percebida muitas vezes no momento em que o mesmo começa a despertar do estado anestésico e a se comunicar. O processo de enfermagem e o uso de escalas e protocolos embasados em teoria cientifica auxiliam durante esse processo e permite que sejam realizadas ações de enfermagem não somente para o diagnóstico, mas também para prevenção e controle do delirium ${ }^{(2)}$.
Diante do exposto, a questão norteadora deste estudo é: "Quais as principais ações da equipe de enfermagem para a prevenção e controle de delirium em pacientes pós-operatório de cirurgia cardíaca?"

Portanto, o objetivo do trabalho é analisar as ações de enfermagem no controle e prevenção do delirium em pacientes pós-operatório de cirurgia cardíaca apresentados na literatura.

\section{MÉTODO}

Trata-se de um estudo de revisão integrativa da literatura. É conduzido através de seis etapas, onde a primeira corresponde a identificação e elaboração da pergunta norteadora, a segunda etapa é o estabelecimento de critérios para inclusão e exclusão de estudos, a terceira, definição das informações a serem extraídas dos estudos selecionados, a quarta, avaliação dos estudos incluídos na revisão integrativa, a quinta etapa consiste na interpretação dos resultados e, a sexta e última etapa, a apresentação da revisão e síntese do conhecimento ${ }^{(7)}$.

Para elaboração da questão norteadora foi utilizada a estratégia PICO, que representa um acrônimo de Pacients (paciente), Intervention (intervenção), Comparison (comparação) e Outcomes (desfecho), onde (P) corresponde a delirium em pacientes pós operatório de cirurgia cardíaca, (I) a ações de enfermagem, (C) não se aplica ao estudo e $(\mathrm{O})$ ao controle e prevenção ${ }^{(8)}$.

A busca e seleção dos artigos foi realizada no portal da Biblioteca Virtual em Saúde (BVS) utilizando as bases de dados Literatura Latino-Americana e do Caribe em Ciências da Saúde (LILACS), Medical Literature Analysis and Retrievel System Online (MEDLINE), Base de Dados de Enfermagem (BDENF), Índice Bibliográfico Espanhol de Ciências de Saúde (IBECS), Literatura em Ciências da Saúde dos países do Caribe (MEDCARIB), Centro Nacional de Informação de Ciências Médicas de Cuba (CUMED), Secretaria Estadual de Saúde de São Paulo e coleciona SUS, 
através do cruzamento dos descritores de saúde (DeCS) "delirium", "pós operatório de cirurgia cardíaca", "pacientes pós operatório de cirurgia cardíaca", "enfermagem", "cuidados de enfermagem", "prevenção de delirium", "controle do delirium", "complicações pós operatório de cirurgia cardíaca" e "cirurgia cardíaca", juntamente com os seus sinônimos e versões em inglês e espanhol.

Os critérios de inclusão foram trabaIhos relacionados ao tema escritos em português, inglês e espanhol completos e disponível online, nos últimos 10 anos. A Figura 1 descreve o percurso realizado para a identificação, a inclusão e a exclusão dos artigos, segundo a base de dados consultadas.

A coleta de dados foi realizada nos meses de outubro a novembro de 2020, por meio de um instrumento estruturado e validado por Ursi (2005), que apresenta as seguintes informações: identificação do artigo original, características metodológicas do estudo, avaliação do rigor metodológico, das intervenções mensuradas e dos resultados encontrados ${ }^{(9)}$. A validação da revisão ocorreu por meio de dois pesquisadores no intuito de aumentar a confiabilidade do estudo.

\section{RESULTADOS}

Foram incluídos no estudo nove ar- tigos que se adequaram aos critérios de inclusão estabelecidos. Ao avaliarmos esses estudos podemos observar que os anos de publicação dos artigos variaram de 2009 a 2020. Entre as profissões dos responsáveis pela autoria dos estudos, a maioria foram realizados por enfermeiros, sendo somente um estudo por médicos. Quanto ao idioma, predominou-se o inglês, sendo seis estudos em inglês, dois em português e um em espanhol. Dentre os países onde foram realizadas as pesquisas, o resultado foi bastante diversificado, sendo um estudo em cada país, entre eles o Irã, Grécia, Holanda, Suécia e Espanha, e os únicos em que duas pesquisas foram realizadas foi no Brasil e o Estados Unidos.

O tipo de estudo mais prevalente foi o estudo prospectivo (44,5\%), seguido do descritivo exploratório (22,2\%), do ensaio clínico randomizado $(22,2 \%)$ e do retrospectivo (11,1\%). Quando ao nível de evidência dos estudos, baseada na categorização da Agency for Healthcare Research and Quality (AHRQ) dos Estados Unidos da América, a classificação variou de um a quatro, predominando o nível de evidência três $(50 \%)$ entre as pesquisas ${ }^{(10)}$.

Em relação aos objetivos e aos assuntos principais dessas pesquisas, a grande maioria explora a avaliação de escalas para a detecção do delirium, além de também ter estudos sobre as ações rea-

Figura 1: Percurso para busca e seleção dos estudos nas bases de dados.

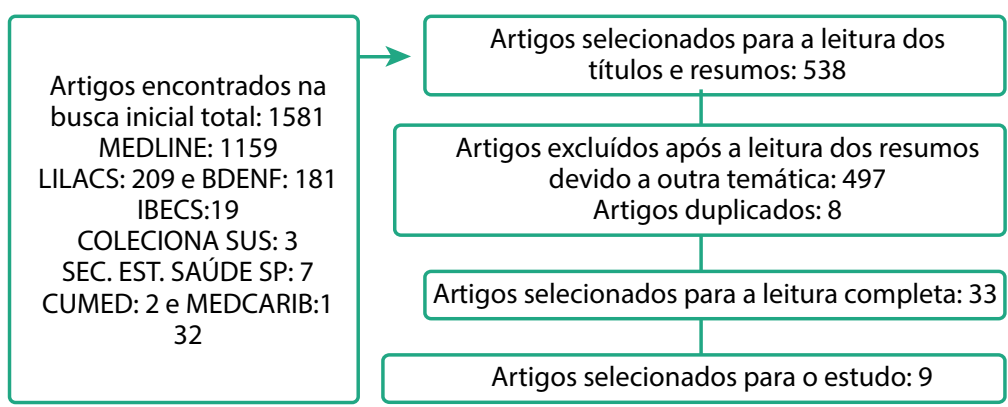

Fonte: Dados da pesquisa, 2020 lizadas para a prevenção, fatores desencadeantes do delirium e ações de enfermagem para o manejo e controle dessa complicação.

\section{DISCUSSÃO}

O delirium não possui uma causa especifica, sabe-se que alguns fatores predispõem a presença dessa patologia. Em um artigo publicado em 2015 foi realizado uma pesquisa em prontuários, sendo possível verificar que pacientes que foram submetidos a cirurgias cardíacas e que apresentaram o delirium no pós-operatório, tiveram um tempo maior de cirurgia e de CEC, perda de sangue aumentada e tinham idade maior que 70 anos. Entre as comorbidades apresentadas nos pacientes que tiveram delirium foram hipertensão, dislipidemia, diabetes, tabagismo, insuficiência cardíaca, doença renal, e arritmias e o uso de medicamentos como zolpidem e benzodiazepínicos ${ }^{(11)}$.

Em estudo recente com pacientes em pós-operatório de cirurgias cardíacas foi possível analisar que os pacientes submetidos a cirurgia de revascularização miocárdica e trocas valvares e que tiveram um tempo maior de CEC e de isquemia durante a CEC apresentaram uma incidência maior de delirium. O delirium pode carretar várias complicações como sedação e ventilação mecânica por maior tempo, tempo maior de internação e risco aumentando de quedas, por isso é necessário que sejam realizados o diagnóstico e reconhecimento do delirium precocemente $^{(12)}$.

Para prever o aparecimento do delirium um estudo traz que é necessário que se avalie a presença dos fatores de risco para o delirium que o paciente apresenta e, caso o paciente apresente três ou mais fatores ele é considerado de alto risco para o desenvolvimento do delirium ${ }^{(13)}$.

Sobre as ações para a prevenção do delirium, um dos focos de um dos estudos foi a comunicação e participação familiar, em que no segundo dia de cirurgia cardíaca e após o término da ventilação 
mecânica e ainda inserido na unidade de terapia intensiva, esse paciente receba, no período da manhã, uma visita familiar estruturada por cerca de 30 a 40 minutos, em que o familiar, antes da visita, é orientado pela equipe de enfermagem sobre o que deve abordar durante a visita, tais como orientar sobre sua localização atual, data e hora, o motivo de estar internado, além de oferecer ao paciente próteses como óculos, aparelho auditivo, caso ele faça uso, para uma melhor comunicação e auto identificação ${ }^{(14)}$.

Foi possível observar que após essa ação, a incidência de delirium diminuiu cerca de $21 \%$. Também foi citado que muitos pacientes apresentavam o delírio no fim da tarde, portanto, os autores sugeriram a visita familiar também neste período ${ }^{(14)}$.

Já outro estudo realizado somente com enfermeiros que atuam em terapia intensiva, apresenta as principais ações realizadas para prevenir e detectar o delirium. Dentre as ações estão a visita familiar por tempo estendido, ações para se evitar o uso indiscriminado de contenção mecânica, controle do ambiente quanto a ruídos e luminosidade. Em relação ao uso de ferramentas para o diagnóstico e controle do delirium, muitos investigados responderam que não utilizam pois não fazem parte do protocolo local ou pelo fato de não conhecerem nenhuma estratégia ${ }^{(15)}$.

Em relação ao manejo do delirium, é citado por um estudo que uma das principais ações não farmacológicas é conhecer sobre o delirium, seus tipos e principais sintomas. Também é importante realizar orientação verbal em tempo e espaço para o paciente utilizando de dispositivos auxiliares para localização de espaço e tempo, como relógios, televisores, janelas, dentre outros. A participação da família no processo de reorientação mais uma vez é citada neste trabalho ${ }^{(16)}$.

É necessário que a contenção mecânica somente seja utilizada quando for o único meio possível para prevenção de danos. Reduzir o máximo possível a presença de ruídos, adequar e organizar os horários das medicações e os proce- dimentos com o intuito de não perturbar o período de sono do paciente, reduzir a restrição da mobilidade, estimular a mobilização precoce, importante para manter a atividade muscular, passiva ou ativa, minimizando-se a atrofia, diminuindo dias de ventilação mecânica e o tempo de permanência na unidade. Além disso, é necessário que seja realizado o reconhecimento precoce do delirium. Uma das escalas mais citadas, é a Confusion Assessment Method (CAM), onde são avaliadas quatro características, sendo a primeira a mudança aguda do estado mental ou curso flutuante do mesmo, seguida da falta de atenção, o pensamento desorganizado e o nível de consciência alterado ${ }^{(16)}$.

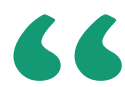

\section{Foi possível observar que após essa ação, a incidência de delirium diminuiu cerca de $21 \%$. Também foi citado} que muitos pacientes apresentavam o delírio no fim da tarde, portanto, os autores sugeriram a visita familiar também neste período

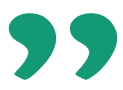

A escala Delirium Observation Screening (DOS) foi projetada para medir os primeiros sintomas de delirium que os enfermeiros podem avaliar durante os cuidados regulares de enfermagem. Nesta escala são avaliados 13 itens. Uma pontuação total de três ou mais pontos indica a presença do delirium ${ }^{(17)}$.

Nesse estudo foi possível verificar que essa escala possui uma alta sensibilidade especificidade, de $100 \%$ e $96,6 \%$, respectivamente. Isso torna a escala DOS um instrumento útil, válido e amigável para determinar o delirium em pacientes que foram submetidos à cirurgia cardíaca e pode ser realizado tanto por enfermeiros quanto por médicos quando treinados. Porém, de acordo com o estudo, a escala DOS, em pacientes que estão intubados pode não ser um instrumento válido, nesses casos recomenda-se utilizar outras escalas como a CAM-ICU (17).

Outro estudo avaliou o uso da escala Nursing Delirium Screening Scale (Nu-DESC ou NDSS) por enfermeiros em pacientes em pós-operatório de cirurgias cardíacas. Essa escala não requer a participação do paciente e são avaliados cinco itens. Esta escala requer uma pontuação maior que dois para o diagnóstico de delirium e pode trazer uma pontuação máxima de 10 pontos, indicando um delirium mais grave ${ }^{(18)}$.

De acordo com Lingehall et al (2012) essa escala tem baixa sensibilidade e especificidade alta, além disso a NU-DESC não detecta todos os tipos de delirium principalmente o hipoativo, que é o predominante em cirurgias cardíacas, sendo necessário que se realize um teste cognitivo para que seja feita o diagnóstico correto. Portanto, a NU-DESC não pode diagnosticar sozinha o delirium ${ }^{(18)}$.

Outro trabalho comparou o uso da NDSS com a escala Confusion Assessment Method for Intensive Care Units (CAM-ICU). Esta escala atualmente é recomendada internacionalmente para o reconhecimento e diagnóstico do delirium em unidades com pacientes críticos. Antes de se realizar a avaliação da CAM-ICU é necessário que se realize uma avaliação prévia do paciente com a escala de RASS (Escala de Agitação- seda- 
ção de Richmond) para pacientes em uso de sedação. E, se o paciente apresentar uma pontuação entre $-3 \mathrm{a}+4$, ele poderá ser examinado para delirium ${ }^{(19)}$.

Na CAM-ICU são avaliadas quatros características: $1^{\circ}$ Flutuação do estado mental basal, $2^{\circ}$ intenção, $3^{\circ}$ alteração do nível de consciência e $4^{\circ}$ pensamento desorganizado, o delirium é diagnosticado quando as características 1 e 2 são positivas e as características 3 ou 4 estão presentes ${ }^{(19)}$.

Comparando-se as duas escalas, verificou-se que a utilização da NDSS sobre a CAM-ICU não seria indicada no pós-operatório imediato de cirurgia cardíaca, pois a NDSS superestimaria sua incidência em até $8 \%$, o que levaria a um tratamento excessivo inadequado, nesses casos a CAM-ICU seria a melhor escolha para realizar o diagnóstico de delirium. A NDSS é de fácil aplicação, porém ela é melhor utilizada em enfermarias ${ }^{(19)}$.

Atualmente a escala que melhor detecta o delirium e a mais recomendada em protocolos é a CAM-ICU, ela pode ser utilizada por qualquer profissional de saúde, porém essa escala ainda é pouco utilizada por falta conhecimento dos profissionais, falta de treinamentos nas instituições e dificuldade de aplicação nos pacientes internados nessas unidades ${ }^{(19)}$.

\section{CONCLUSÃO}

Neste estudo, ficou evidenciado através dos artigos selecionados, que o delirium é uma complicação que não possui uma causa específica, porém alguns fatores podem influenciar nesse aparecimento, como o tempo prolongado de cirurgia e CEC, tipo de cirurgia, idade e as comorbidades que o paciente possui e para prever o aparecimento do delirium é necessário que se avalie a presença desses fatores. A enfermagem é essencial para realizar ações preventivas e reconhecer e diagnosticar o delirium em pacientes pós-operatório de cirurgia cardíaca. Muitas ações, principalmente de prevenção são realizadas, e a comunicação e pre- sença da família é fundamental para se prevenir o aparecimento dessa patologia.

Além disso, foi possível verificar que existem algumas escalas de fácil aplicação para realizar a identificação precoce do delirium, sendo a CAM-ICU a escala mais sugerida e de melhor aplicação, entre as escalas apresentadas pela literatura, porém elas são pouco conhecidas e utilizadas pelos os profissionais, sendo necessário que se disseminem as já existentes, para que elas sejam mais empregadas e consequentemente diminuir as complicações que esses pacientes possam apresentar devido a presença do delirium.

Espera-se que o presente estudo possa contribuir para uma reflexão sobre a atuação e as ações da enfermagem para o manejo e controle do delirium nesses pacientes e que essas intervenções sejam mais realizadas. Observou-se que apesar de haver estudos relacionadas ao tema, ainda é necessário que haja mais investimentos na condução de pesquisas futuras sobre a problemática.

\section{Referências}

1. Wacker $P$, Nunes $P V$, Forlenza 0 . Delirium: uma perspectiva histórica. Rev Psiquiatr Clín. 2018; 32 (3): 97-103.

2. Bastos AS, Beccaria LM, Silva DC, Barbosa TP. Identificação de delirium e delirium subsindromático em pacientes de terapia intensiva. Rev Bras Enf. 2019; 72 (2): 463-467..

3. Bisinotto FMB, Silveira LAM, Silva RO, Martins LB. Delirium pós-operatório no idoso. Onde estamos?. Rev Med Minas Gerais. 2017; 27 Supl 2: S52-S66. 4. Lourenço BC, Narciso AC, Amorim EH, Almeida FCA, Cruz RAO. Processo do cuidar em enfermagem no perioperatório de cirurgia cardíaca. Braz. J. Surg. Clin. Res. 2013; 22: 2858-2866.

5. Neves AFC, Giustina KPD. Estudo sobre delirium em pós-operatório de cirurgia cardíaca, em um hospital de alta complexidade da região sul de Santa Catarina [monografia disponível da internet]. [Santa Catarina] UNIEDU; 2014 [Acesso em: 06 set. 2020]. Disponível em: http://www.uniedu.sed.sc.gov.br/ wp-content/uploads/2014/04/amanda_fernandes_candido_neves.pdf.

6. Oliveira FRA. Incidência, fatores preditores e consequências do delirium no pós operatório de cirurgia cardíaca [Tese disponível na internet]. [São Paulo] Faculdade de Medicina da universidade de São Paulo; 2015 [Acesso em: 05 set. 2020]. Disponivel em: https://www.teses.usp.br/teses/disponiveis/5/5131/ tde-11092015-160812/pt-br.php.

7. Souza MT, Silva MD, Carvalho R. Revisão integrativa: o que é e como fazer. Einstein. 2010; 8 (1): 102-106.

8. Santos CMC, Pimenta CAM, Nobre, MRC. A estratégia pico para a construção da pergunta de pesquisa e busca de evidências. Rev Latino-am Enfermage. 2007 mai; 15(3).

9.Ursi ES, Galvão CM. Prevenção de lesões de pele no perioperatório: revisão integrativa da literatura. Rev Latino-am Enfermagem. 2006; 14(1):124-31.

10. Galvão CM, Sawada NO, Mendes IAC. A busca das melhores evidências.
Rev Esc Enferm USP. 2003; 37(4): 43-50.

11. Mangusan RF, Hooper V, Travis L. Outcomes associated with postoperative delirium after cardiac surgery. Am J Crit Care. 2015; 24 (2): 156-163.

12. Theologou S, Giakoumidaki K, Charitos C. Perioperative predictors of delirium and incidence factors in adult patients post cardiac surgery. Pragmat $\mathrm{Obs}$ Res. 2018; 9: 11-19.

13. Marra A, Ely EW , Pandharipande PP, Patel MB. The ABCDEF Bundle in Critical Care. Crit Care Clin. 2017 abr.; 33(2): 225-243.

14. Babadi ME, Shokrollahi N, Mehrabi T. Effect of family-patient communication on the incidence of delirium. Iran J Nurs Midwifery Res. 2017 jul/aug; 22 (4): 327-31.

15. Oliveira KP, Picanço CM, Oliveira AR, Assis YIS, Souza ACF, Ribeiro AG. Estratégias utilizadas por enfermeiras para minimizar a ocorrência de delirium em pacientes críticos. Rev. Enferm. UFSM. 2020; 10:1-18.

16. Eberle CC, Santos AA, Macedo Junior LJJ, Martins JB. 0 Manejo Não Farmacológico do Delirium Sob a Ótica de Enfermeiros de uma Unidade de Terapia Intensiva Adulto. J. res. fundam. care. Online. 2019 out/dez; 11(5):1242-1249. 17. Koster S, Hensens AG, Oosterveld FGJ, Wijma A, Palen JVD. The delirium observation screening scale recognizes delirium early after cardiac surgery. Eur. J. Cardiovasc. 2009; 8:309-314.

18. Lingehall HC, Smulter N, Engstrom KG, Gustafson Y, Olofsson B. Validation of the Swedish version of the Nursing Delirium Screening Scale used in patients 70 years and older undergoing cardiac surgery. J. Clin. Nurs. 2013; 22:2858-2866.

19. Peréz AA, Poza SC, Catalán CC, Fernández AG, Vidal YJN. Valoración de la concordancia entre la escala CAM-ICU y la nursing delirium screening scale en el postoperatorio de cirugía cardiaca en una unidad de críticos. Enferm intensiva. 2014; 25(3): 100-106 